Admissions. - M. Otto Budy, libraire-éditeur, 11, Carlstrasse, Berlin N.-W., 40 (Allemagne). Bibliographie entomologique.

- M. Mario-Sanchez Roig, calzada del Cerro, no 827, La Havane (Cuba). Arachnides, Lépidoptères.

Démission. - M. L. Boutan (d'Hanoï).

Radiations. - M. Lahaussors, Trésorier, propose à la Société de rayer de la liste de ses membres un certain nombre de personnes qui ne payent plus leurs cotisations depuis plusieurs années et ne répondent même pas aux lettres de réclamation qui leur sont envoyées.

La Société prononce la radiation de MM. G. BeAulieu, de Montréal, - M. Сholodkowsky, de St-Pétersbourg, - A. Joanin, de Châtillonsous-Bagneux, - N. Naçonow, de St-Pétersbourg, - G. D'OlsoufiefF, de Volhynie (Russie), - A. Ponselle, de Paris, - J. Roguier, d'Aubervilliers, - N. Schawroff, de Tiflis (Russie), - E. Tisson de T'horas, d'Aymargues (Gard), - C. Tourte-Hawkins, de Verdun (Meuse), el de la Bibliothèque de L'Université de Rennes.

\title{
Communications.
}

\section{Description de deux espèces nouvelles \\ appartenant au genre Rhatymoscelis Thoms [CoL. Cerambycidae]}

par E. GounelLe.

Le genre Rhatymoscelis n'est représenté jușqu'ici que par une seule espèce originaire du Mexique, R. Haldemani Thoms.

En voici deux autres ayant le Brésil pour habitat. Je dédie la première au savant directeur du musée de Sao Paulo et la seconde au R. Père Donme, ancien professeur du collège du Caraça, Minas, dont feu l'abbé DAvid, qui m'en a fait don, l'avait reçue jadis.

Rhatymoscelis Iheringi, n. sp. - Atro cyanea, subtiliter griseopubescens, elytrorum maculis duabus minutis, abdominis segmento primo basi tarsisque posticis pallide luteis, alis brunne-fuliginosis; caput punctato-coriaceum, inter antennas concavum, infra oculos late. raliter utrinque dilatatum, fronte sulcata et carinulis duabus obliquis, 
arcuatis, a tuberibus antenniferis excurrentibus munita, processu jugulari dente valido, obtuso subtus armato, vertice basi transversim tumido; antennae (ㅇ?) capite et thorace simul sumptis duplo paulo longiores, 11-articulatae, art. ultimo appendiculato, scapo brevissimo, obconico, art. 3 sequentibus paulo breviore, art. 1-10 subaequalibus, intus apice breviter serratis; thorax paulo transversus, subquadratus, lateribus rectis, parallelis, subtilissime punctato-coriaceus, supra inaequalis, scilicet tuberculis quatuor debilibus, duobus juxta basim, duobus anticis paululum majoribus carinula obsoleta leniter biarcuata transversim con. junctis instructus; scutellum magnum, triangulare, in medio depressum; elytra brevissima, metasterni medium paulo superantia, squamas duas simulantia, a scutello usque ad apicem obliquiter subarcuatim dehiscentia, subtilissime punctulata, apice obtuse acuminata, singulatim bicostata, costis arcuatis, postice conjunctis, humeris rotundatis glabris, laevibus, valde prominentibus; coxae anticae obconicae, exsertae, extus angulatae, acetabula media

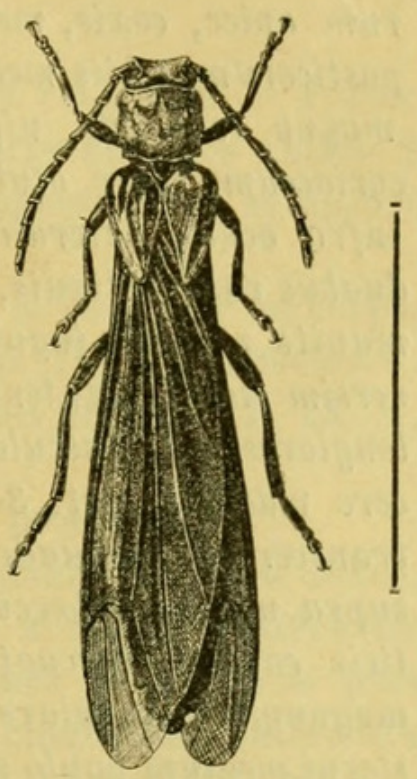

Fig. 1. - Rhatymoscelis Iheringi Gounelle. extus valde hiantia; pedes breves, debiles, postici anticis longiores, metasternum amplum, episternis cuneatis; abdomen elongatissimum, molle, cupite, thorace elytrisque simul sumptis duplo longius, basi subpetiolatum, cylindratum, postice gradatim paulo dilatatum et depressum, segmento primo caeteris singulis multo longiore. - Long. : 21-30 mill. - 3 exemplaires.

Deux d'entre eux ont été capturés dans l'État de Saô Paulo; l'un appartient au musée Paulista; l'autre trouvé par moi à la Fazenda Cerqueira Cesar, Val du Rio Pardo, se trouve dans ma collection ainsi qu'un troisième individu provenant de l'État de Minas Geraës.

Ce dernier, un peu différent des exemplaires de Saô Paulo, a les tarses postéricurs noirâtres comme ceux des pattes antérieures et la tache jaunâtre des élytres, beaucoup plus grande, recouvre presque entièrement le disque de ces organes.

D'après la formule du genre Rhatymoscelis, le ơ a douze articles aux antennes; nous venons de voir que les trois individus types de $R$. Theringi n'en ont que onze, le dernier étant appendiculé, il y a donc tout lieu de supposer que ce sont des f. 
Par sa coloration d'un bleu noir légèrement métallique, cette nouvelle espèce se distingue à première vue de $\boldsymbol{R}$. Haldemani.

Rhatymoscelis Dormei, n. sp. - Testacea, subtiliter flaro-pubescens, capite, antennis, thoracis disco fasciaque infera, scutello, elytrorum apice, coxis, meso- et metasternis, claia femorum mediorum et posticorum, tibiis mediis et posticis apice, tarsis abdominisque segmentis magna ex parte, nigris, alis sordide flaro-testaceis; caput punctatocoriaceum, inter oculos et antennas concarum et profunde sulcatum, infra oculos lateraliter utrinque paulo dilatatum, fronte carinulis duabus valde obliquis, arcuatis, a tuberibus antenniferis excurrentibus munita, processu jugulari dente minuto subtus armato, vertice basi transversim elevato; antennae ơ capite thoraceque simul sumptis vix duplo longiores, crassiusculae, 12-articulatae (art. ultimus praecedenti coalescere videtur), art. 3-11 intus apice breviter serratis; thorax faulo transversus, subquadratus, lateribus rectis, parallelis, haud punctatus, supra nitidus tuberculisque quatuor, duobus juxta basim, duotus anticis carina biarcuata transiersim conjunctis instructus; scutellum magnum, triangulare, in medio depressum; elytra brevissima, metasterni medium paulo superantia, squamas duas simulantia, a scutello ad apicem obliquiter dehiscentia, subtilissime coriacea, apice obtuse angulata, singulatim obsolete unicostata, humeris rotundatis, valde prominentibus, sublaevilus; coxae anticae obconicae, exsertae, exlus angulatae; acetabula media extus hiantia, pedes breves, postici anticis longiores, femoribus anticis et mediis valde compressis; metasternum amplum, episternis cuneatis; abdomen elongatissimum, capite thorace elytrisque simul sumptis sesquilongius, molle, subcylindratum, postice arcuatum, segmento primo caeteris singulis multo longiore. - Long. : 14 mill. 1 exemplaire.

État de Minas Geraës; ma collection.

Cette espèce se distingue de la précédente par sa petite taille, sa coloration, son thorax non ponctué, ses élytres moins falciformes, etc. 


\section{$2 \mathrm{BHL}$ Biodiversity Heritage Library}

Gounelle, Pierre-Émile. 1910. "Description de deux espèces nouvelles appartenant au genre Rhatymoscelis Thoms [Col. Cerambycidae]." Bulletin de la Société entomologique de France 1910, 46-48. https://doi.org/10.5962/bhl.part.4657.

View This Item Online: https://www.biodiversitylibrary.org/item/38239

DOI: https://doi.org/10.5962/bhl.part.4657

Permalink: https://www.biodiversitylibrary.org/partpdf/4657

\section{Holding Institution}

Smithsonian Libraries

\section{Sponsored by}

Smithsonian

\section{Copyright \& Reuse}

Copyright Status: NOT_IN_COPYRIGHT

This document was created from content at the Biodiversity Heritage Library, the world's largest open access digital library for biodiversity literature and archives. Visit BHL at https://www.biodiversitylibrary.org. 